\title{
Compound pathogenic mutation in the USH2A gene in Chinese RP families detected by whole-exome sequencing
}

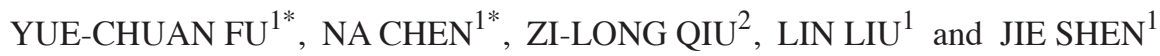 \\ ${ }^{1}$ Department of Ophthalmology, The Shanghai Renji Hospital, Shanghai Jiaotong University School of Medicine, \\ Shanghai 200120; ${ }^{2}$ Institute of Neuroscience, CAS Center for Excellence in Brain Science and Intelligence Technology, \\ Chinese Academy of Sciences, Shanghai 200031, P.R. China
}

Received March 10, 2018; Accepted September 4, 2018

DOI: $10.3892 / \mathrm{mmr} .2018 .9530$

\begin{abstract}
Retinitis pigmentosa (RP) is a common form of inherited retinal degeneration that causes progressive loss of vision or adult blindness, characterized by the impairment of rod and cone photoreceptors. At present, mutations in $>60$ pathogenic genes have been confirmed to cause RP. The predominant modes of inheritance are autosomal dominant, autosomal recessive and X-linked. In addition, other modes of inheritance, including digenic or mitochondrial inheritance, have been reported. In previous decades, with the development of sequencing techniques, significant advances in identifying novel RP pathogenic genes and screening mutations have been made. In the present study, whole-exome sequencing was performed on samples from two Chinese pedigrees diagnosed with RP. A compound heterozygous mutation in the gene usherin $2 \mathrm{~A}$ (USH2A; c.6,485+5G>A/c.11,156G $>\mathrm{A}$ ) and a heterozygous $\mathrm{X}$-linked mutation in the gene retinitis pigmentosa 2 (RP2) ARL3 GTPase-activating protein (RP2; c.358C $>$ T) were identified by Sanger sequencing and co-segregation analysis, of which the pathogenic mutation (c.6,485+5G $>$ A) in USH2A has not been previously reported among Chinese patients. The findings of the present study may expand on current knowledge of RP among the Chinese population, providing essential assistance in the molecular diagnosis and screening of RP, and promoting further investigation of the pathogenesis of RP.
\end{abstract}

Correspondence to: Professor Jie Shen or Professor Lin Liu, Department of Ophthalmology, The Shanghai Renji Hospital, Shanghai Jiaotong University School of Medicine, 160 Pujian Road, Shanghai 200120, P.R. China

E-mail: jshenrenji@163.com

E-mail: liulin1963rj@126.com

*Contributed equally

Key words: retinitis pigmentosa, whole-exome sequencing, gene mutation, RP2, USH2A

\section{Introduction}

Retinitis pigmentosa (RP) is an inherited retinal dystrophy that causes adult blindness, and is characterized by the degeneration of rod and cone photoreceptors. Typical symptoms include night blindness at an early age and progressive constriction of the mid-peripheral visual field. Patients with RP eventually become completely blind. There are three major modes of inheritance: Autosomal dominant (ADRP), autosomal recessive (ARRP) and X-linked (XLRP) (1). In addition, other modes of inheritance, including digenic or mitochondrial inheritance, have been reported $(2,3)$.

$\mathrm{RP}$ can also occur as a syndrome accompanying other diseases. Usher syndrome is the most frequent form of syndromic RP, characterized by RP accompanied by sensorineural hearing loss. Usher syndrome can be divided into three types based on the assessment of clinical features.

Given the complexity and perniciousness of RP, molecular diagnosis is a potentially effective method of analysis that can be used to diagnose RP and assess disease prognosis. Although the traditional method, Sanger sequencing, can be a valid strategy used to detect genetic abnormities, analyzing a large number of candidate genes with this method is expensive (4). With the advance of whole-exome sequencing technology, determining full-coding regions has become faster (5). Whole-exome sequencing is particularly useful in the detection of novel disease-causing genes (6).

In the present study, one Chinese pedigree with ARRP and one Chinese pedigree with XLRP were investigated. Whole-exome sequencing was performed on the affected patients, identifying a compound heterozygous mutation in usherin (USH2A) and a heterozygous X-linked mutation in ARL3 GTPase-activating protein (RP2). The mutations co-segregated with the diseases in the pedigrees and, to the best of our knowledge, the pathogenic mutation (c. $6,485+5 \mathrm{G}>\mathrm{A}$ ) in USH2A has not been reported previously among Chinese patients.

\section{Materials and methods}

Subjects and clinical assessment. The present study involved seven individuals from two Chinese families with typical clinical features of RP. The two pedigrees were labeled Family 
$\mathrm{D}$ and Family $\mathrm{H}$ and the pedigrees of the two families are shown in Fig. 1A and B. The present study was performed in accordance with the tenets of the Declaration of Helsinki and the ethical guidelines of the Shanghai Renji Hospital, Shanghai Jiaotong University School of Medicine (Shanghai, China). Written informed consent was obtained from all participants. The clinical examinations performed to diagnose $\mathrm{RP}$ predominantly included assessing the best-corrected visual acuity (BCVA) using a Snellen chart, capturing images of each fundus, assessing computerized test of central and peripheral visual fields, and optical coherence tomography (OCT).

DNA extraction. Peripheral blood samples were obtained from all subjects, and used for DNA extraction using a kit (Qiagen, Shanghai) according to the manufacturer's protocols. DNA yield was quantified with NanoDrop One (Thermo Fisher Scientific, Inc., Wilmington, DE, USA) and $1.5 \mu \mathrm{g}$ of DNA was used for the following assay. The DNA samples were stored at $-80^{\circ} \mathrm{C}$ until use.

Exome capture and sequencing. Whole-exome sequencing was performed by Beijing Youle Fusheng Technology Co., Ltd. (Beijing, China). Sample libraries of gDNA were prepared using the TruSeq DNA Sample Preparation kit (Illumina, Inc., San Diego, CA, USA) and the exomic regions were enriched using the Roche Nimblegen SeqCap EZ v5.1 kit (Roche Applied Science, Madison, WI, USA) according to manufacturers' protocols. Paired-end short read sequencing was performed on an Illumina platform with a PE150 read length (Illumina, Inc.).

Identification of pathogenic mutations from exome data. Bioinformatics analyses were performed using an in-house pipeline. Briefly, sequencing reads that passed quality filtering were aligned with the human reference genome, hg19, using the Burrows-Wheeler Aligner program (BWA version 0.7.15) (7). Variants were identified using a combination of the FreeBayes (version 0.9.21) (8), Genome Analysis Toolkit (GATK version 3.8) (9-11), CNVnator (version 0.2.7) (12) and in-house software programs.

The in-house software program, Langya, was used for variant annotation and filtering. The variants were prioritized based on variant frequency, pathogenicity, inheritance pattern and how closely a gene associated with the given phenotype. The variant frequency was obtained from the 1,000 Genomes Project, Single Nucleotide Polymorphism database (http://www.1000genomes.org) and Exome Aggregation Consortium databases (http://exac.broadinstitute. org). Predicted pathogenicity data were obtained using the SNPEff (http://snpeff.sourceforge.net), Ensembl Variant Effect Predictor (http://asia.ensembl.org/info/docs/tools/vep/index. html), Sorting Intolerant From Tolerant, Polymorphism Phenotyping version 2 (http://genetics.bwh.harvard. edu/pph2/) and dbscSNV databases (https://sites.google. com/site/jpopgen/dbNSFP). Gene-phenotype association was characterized from structured resources including the online Mendelian Inheritance in Man database (https://www. omim.org), and unstructured resources, including literature, utilizing natural language processing techniques. The average sequencing depth in Family D was $>100 X$, whereas it was $\sim 85 \mathrm{X}$ in Family $\mathrm{H}$. The average coverage of the two families was $\sim 99.8 \%$.

Sanger sequencing. The candidate variants in USH2A and RP2 identified in the exome data were validated by Sanger sequencing.

\section{Results}

Clinical assessment of families with RP. The clinical features of the affected patients in the two pedigrees are listed in Table I. The male patient D-II-2 suffered from decreased visual acuity and night blindness at the age of 14 years. The best-corrected visual acuity (BCVA) of D-II-2 decreased to light perception as the patient aged. Although the central vision of the affected female individual, D-II-3, was 20/20, the visual field of the eyes of D-II-3 exhibited severe mid-peripheral scotomata due to a coalescence of scotomata. All of the clinical features fit with the diagnostic criteria of RP. The results of fundus examinations revealed that the patients exhibited a typical RP appearance: Pale optic discs, attenuated retinal arterioles and 'bone spicule' pigmentation. The OCT indicated that marked thinning and disruption of the retinal nerve fiber layer, and the retinitis pigment epithelium was evident (Fig. 2A and B). The hearing examinations performed on the patients were normal.

In Family $\mathrm{H}$, a 48-year-old female patient suffered from visual difficulties at night at the age of 20 years; visual field loss and decreased visual acuity progressed markedly from the age of 30 years. In the present study, the BCVA of the patient was finger count $(\mathrm{FC}) / 5 \mathrm{~cm}$. The visual field of the patient's eyes demonstrated mid-peripheral scotomata due to a coalescence of scotomata. The deceased father of the patient had been diagnosed with RP. The patient's son (H-III-1) was diagnosed with RP at the age of 7 years due to night blindness. The visual acuity of H-III-1 decreased significantly following diagnosis. The detailed ophthalmic examination results are shown in the Fig. 3A and B. The fundus examinations indicated that H-III-1 exhibited a typical RP appearance, including 'bone spicule'pigmentary deposits, retinal vessel attenuation and waxy optic discs. The OCT revealed that the retinal thickness was decreased and that the foveal and retinal layers were atrophied. The unaffected individuals exhibited normal phenotypes in all of the ophthalmological examinations.

Pathogenic mutation detection. Whole-exome sequencing was performed on samples from two Chinese families with RP. In Family H, a heterozygous mutation (c.358C $>$ T) in RP2 was identified as a pathogenic mutation, co-segregating with the disease. The c.358C $>\mathrm{T}$ mutation in RP2 was demonstrated to be involved in pathogenic mutation in XLRP. The two patients in Family $\mathrm{H}$ possessed the heterozygous variants, whereas the unaffected individual did not. The two patients in Family D possessed the same compound heterozygous mutation $(\mathrm{c} .6,485+5 \mathrm{G}>\mathrm{A} / \mathrm{c} \cdot 11,156 \mathrm{G}>\mathrm{A})$ in the USH2A gene, which co-segregated with the disease (Table II). The c.6,485+5G $>\mathrm{A}$ mutation is a pathogenic mutation in ARRP, which has not been reported previously in Chinese patients. The results of Sanger sequencing of the validated pathogenic mutations is shown in Fig. 4A and B. Based on the clinical assessments and mutation analyses, the present study concluded that the three 
Table I. Clinical phenotype of the patients in Families D and H.

\begin{tabular}{|c|c|c|c|c|c|c|c|}
\hline Family & ID & Age (years) & Gender & $\begin{array}{l}\text { Age at onset } \\
\text { (years) }\end{array}$ & BCVA & Slit lamp & Refraction \\
\hline \multirow[t]{2}{*}{$\mathrm{D}$} & D-II-2 & 49 & Male & 14 & $\begin{array}{l}\mathrm{R}: \mathrm{LP} / \mathrm{LP} \\
\mathrm{L}: \mathrm{LP} / \mathrm{LP}\end{array}$ & Mild cataract & $\begin{array}{l}\text { R: }-2.50 \mathrm{DS} /-1.75 \mathrm{DC}^{*} 165 \\
\mathrm{~L}:-2.00 \mathrm{DS} /-2.00 \mathrm{DC}^{*} 10\end{array}$ \\
\hline & D-II-3 & 47 & Female & 20 & $\begin{array}{l}\text { R: } 20 / 20 \\
\text { L: } 20 / 20\end{array}$ & Mild cataract & $\begin{array}{l}\text { R: }-7.00 \mathrm{DS} /-1.00 \mathrm{DC}^{*} 160 \\
\mathrm{~L}:-7.25 \mathrm{DS} /-0.75 \mathrm{DC}^{*} 5\end{array}$ \\
\hline \multirow[t]{2}{*}{$\mathrm{H}$} & H-II-2 & 53 & Female & 20 & $\begin{array}{l}\mathrm{R}: \mathrm{FC} / 5 \mathrm{~cm} \\
\mathrm{~L}: 12 / 200\end{array}$ & Mild cataract & $\begin{array}{l}\mathrm{R}:-4.50 \mathrm{DS} /-0.75 \mathrm{DC}^{*} 175 \\
\mathrm{~L}:+0.50 \mathrm{DC} \mathrm{C}^{*} 95\end{array}$ \\
\hline & H-III-1 & 28 & Male & 7 & $\begin{array}{l}\mathrm{R}: 12 / 200 \\
\mathrm{~L}: 12 / 200\end{array}$ & Normal & $\begin{array}{l}\mathrm{R}:-1.25 \mathrm{DS} \\
\mathrm{L}:+1.25 \mathrm{DS}\end{array}$ \\
\hline
\end{tabular}

BCVA, best corrected visual acuity; R, right eye; L, left eye; LP, light perception; FC, finger count; DS, diopter of scope; DC, diopter of cylinder.

Table II. Pathogenic mutations of patients in Families D and H diagnosed with retinitis pigmentosa.

\begin{tabular}{lccccccc}
\hline & \multicolumn{3}{c}{ Chromosome } & & & Amino acid \\
Family & Gene & position & cDNA mutation & Type of mutation & Hom/Het & Inheritance & Origin \\
\hline D & USH2A & $1: 215933077$ & c.6485+5G $>$ A & & Het & Autosomal recessive Father \\
& USH2A & $1: 215933077$ & c.11156G $>$ A & Missense variant & Het & Arg3719Leu & Autosomal recessive Mother \\
H & RP2 & X:46713166 & c.358C $>$ T & Stop-gain & Het & Arg120Ter & X-linked \\
\hline
\end{tabular}

USH2A, usherin; P2, ARL3 GTPase-activating protein; Hom, homozygous; Het, heterozygote.

A

I

II

III

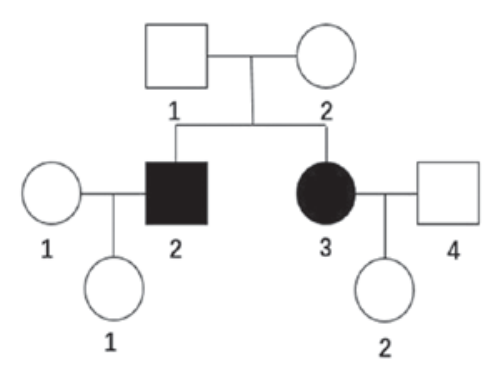

B



Figure 1. Pedigree of two families with retinitis pigmentosa. Pedigree of (A) Family D and (B) Family H. Closed symbols represent affected patients and open symbols represent unaffected individuals. The squares mean male, the circles mean female and the slashes mark deceased.

heterozygous mutations were the pathogenic variants involved in RP in the two families.

\section{Discussion}

In the present study, three mutations associated with RP were reported. In humans, XLRP is one of the most severe forms of RP, with six associated genetic loci. It is estimated that $\sim 20 \%$ of patients with XLRP have pathogenic mutations in RP2. RP2 has been reported as an activator of small GTPase ADP-ribosylation factor like-3, and has been implicated in cell signaling and vesicular transportation by linking the cell membrane with the cilia and cytoskeleton in photoreceptor cells $(13,14)$. Patil et al $(15)$ revealed that RP2 was crucial in photoreceptor development, and distinct mutations in RP2 caused dysfunctions in cone and rod photoreceptors, resulting in a wide-spectrum of clinical phenotypes. In the present study, the c.358C $>\mathrm{T}$ mutation in exon 2 resulted in a truncated RP2 protein through a change of the arginine codon to a nonsense codon. c.358C > T was first reported by Mears et al (16) in 1999. With the development of sequencing techniques, whole-exome sequencing has allowed for significant advances to be made in the identification of novel RP pathogenic genes and screening of mutations. In Family H, the affected male (H-III-1) exhibited more severe clinical features compared with the affected female (H-II-2); H-III-1 had an earlier age of onset, rapid progression of visual field constriction and loss of visual acuity. RP is generally determined by random X-chromosome inactivation, the process responsible for silencing one of the $\mathrm{X}$ chromosomes in females. The X-inactivation center produces 
A a

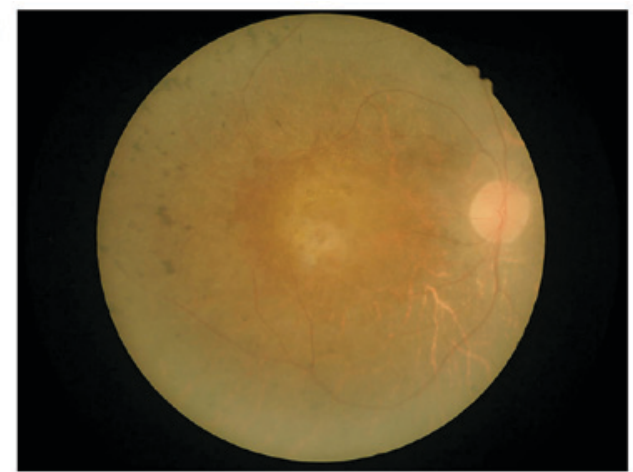

b1

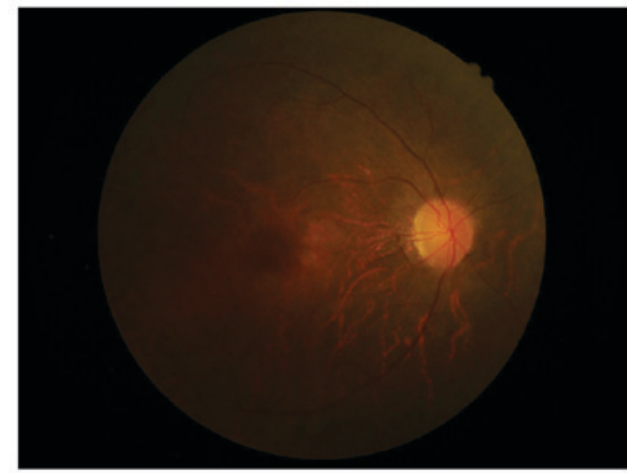

B a

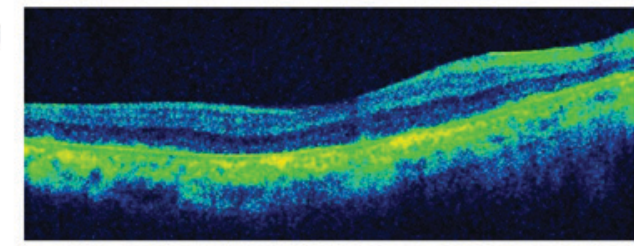

b1

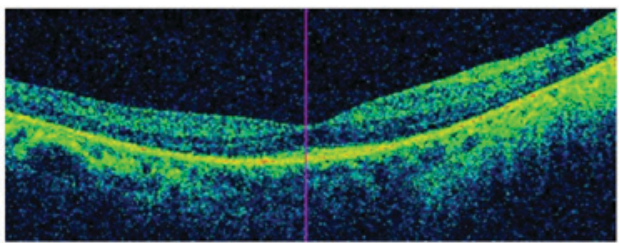

a2

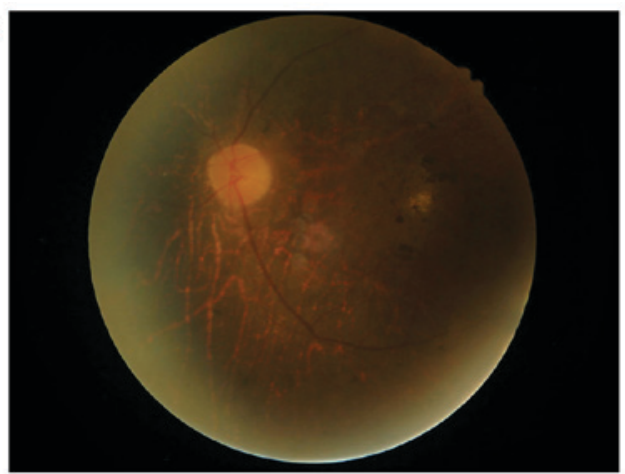

b2

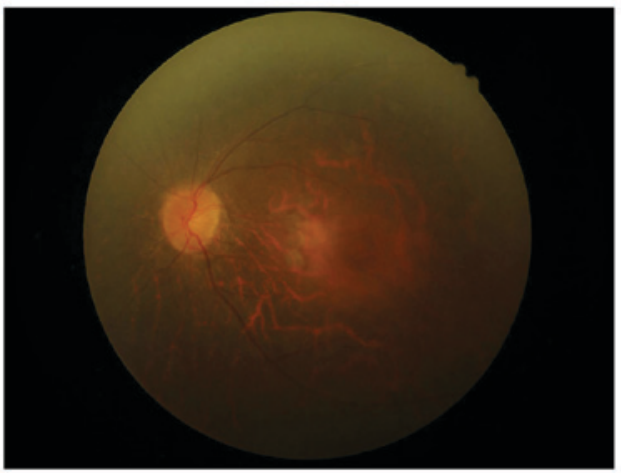

a2

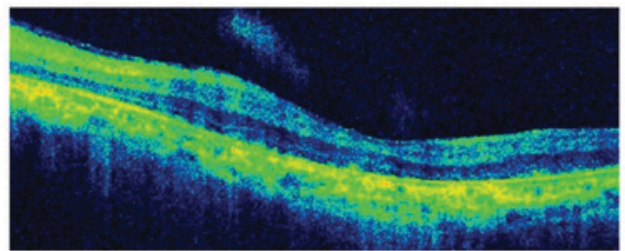

b2

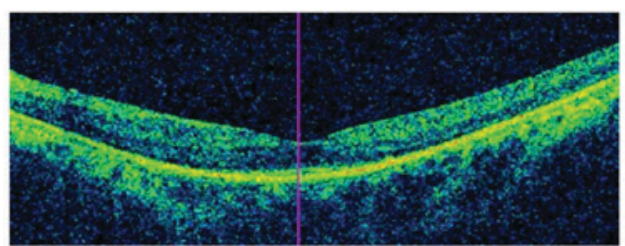

Figure 2. Clinical examination of Family D. (A) Fundus examinations of the (a1) right and (a2) left eyes of D-II-2, and the (b1) right and (b2) left eyes of D-II-3. (B) Optical coherence tomography of the (a1) right and (a2) left eyes of D-II-2, and the (b1) right and (b2) left eyes of D-II-3.

a long functional non-coding RNA, X inactive specific transcript (Xist), which is directly involved in the mediation of chromosome silencing (17). However, the mechanism of Xist silencing requires further investigation.

RP can also occur as a syndrome, which accompanies other diseases. Usher syndrome is the most frequent form of syndromic RP associated with hearing loss (18). It can be divided into three types based on the assessment of clinical features. At present, 500 coding variants in USH2A have been reported to be pathogenic. However, Jacobson et al (19) analyzed photoreceptors and the retinal pigment epithelia of individuals with different mutations in USH2A using OCT, demonstrating consistent results between the individuals with the same USH2A genotype. USH2A is located on chromosome $1 \mathrm{q} 41$ and is the most commonly mutated splice variant of USH2, which consists of two main spliced transcripts. The shorter splice variant consists of 21 exons and encodes a $170 \mathrm{kDa}$ protein. The longer splice variant consists of 72 exons and encodes a $600 \mathrm{kDa}$ protein, which contains a membrane-spanning segment followed by an intracellular
PDZ-binding domain at the C-terminus (20). The expression of the longer splice variant is restricted to photoreceptors and developing cochlear hair cells (21). Liu et al (22) disrupted the expression of USH2A in mice, resulting in the progressive degeneration of photoreceptors and non-progressive hearing impairment, suggesting that USH2A may be essential for the maintenance and development of photoreceptors and cochlear hair cells.

In Family D, D-II-3 and D-II-2 possessed the same compound heterozygous mutation (c.6,485+5G $>\mathrm{A} /$ c. $11,156 \mathrm{G}>\mathrm{A})$ in USH2A. Although the two shared the same mutations, D-II-3 had a central visual field, whereas D-II-2 exhibited almost complete visual field loss. It has been suggested that the same mutations can cause a variety of intrafamilial phenotypes. Previously, it was reported that mutations in USH2A can cause patients to suffer from RP without hearing loss. The missense mutation in USH2A, c.11,156G $>$ A, affects the long splice variant of USH2A, resulting in a substitution of arginine for leucine at protein position 3,719 (23). This change may affect the insertion of the protein into the membrane, 
A

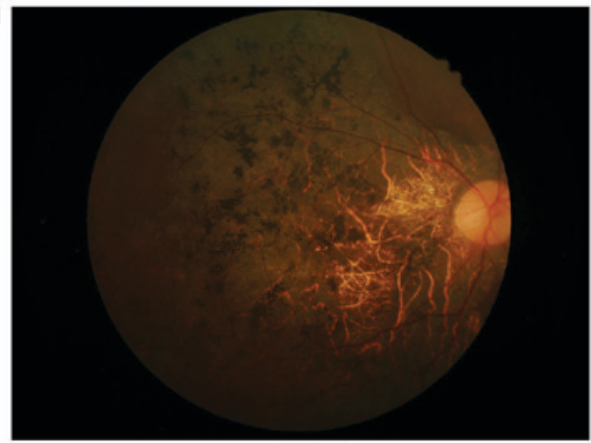

b1



B

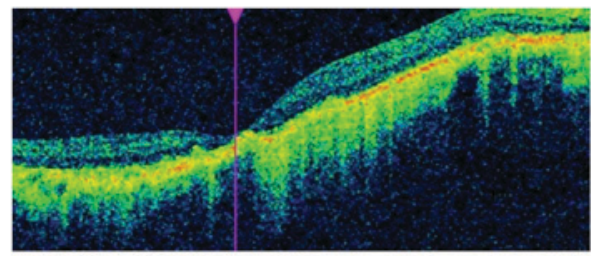

b1

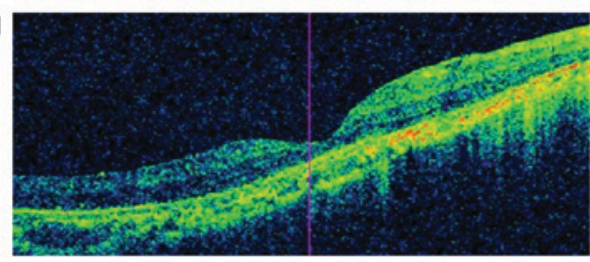

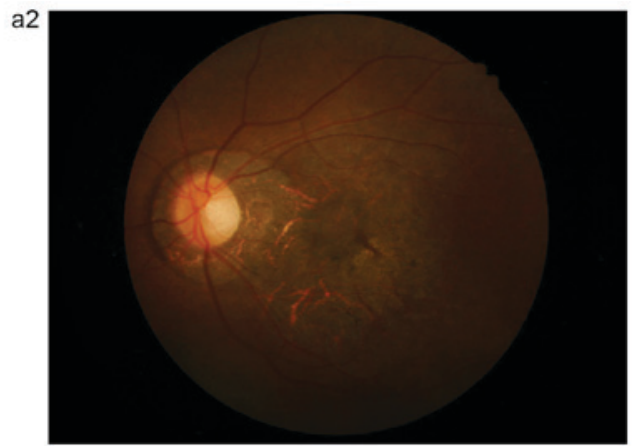

b2

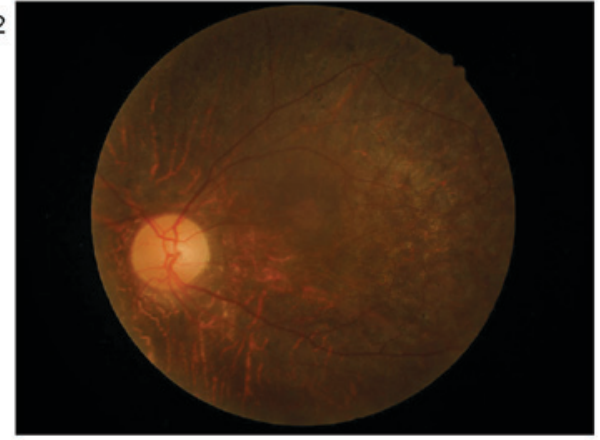

a2

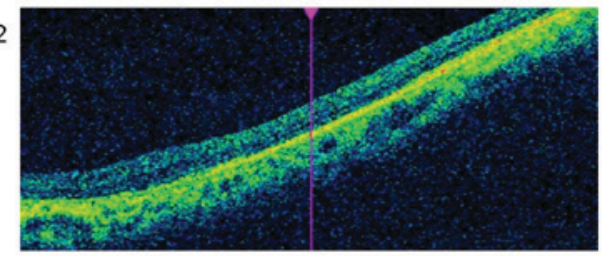

b2

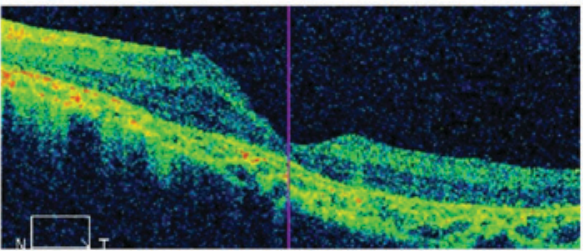

Figure 3. Clinical examination of Family H. (A) Fundus examinations of the (a1) right and (a2) left eyes of H-II-2, and the (b1) right and (b2) left eyes of D-II-3. (B) Optical coherence tomography of the (a1) right and (a2) left eyes of H-II-2, and the (b1) right and (b2) left eyes of H-III-1.

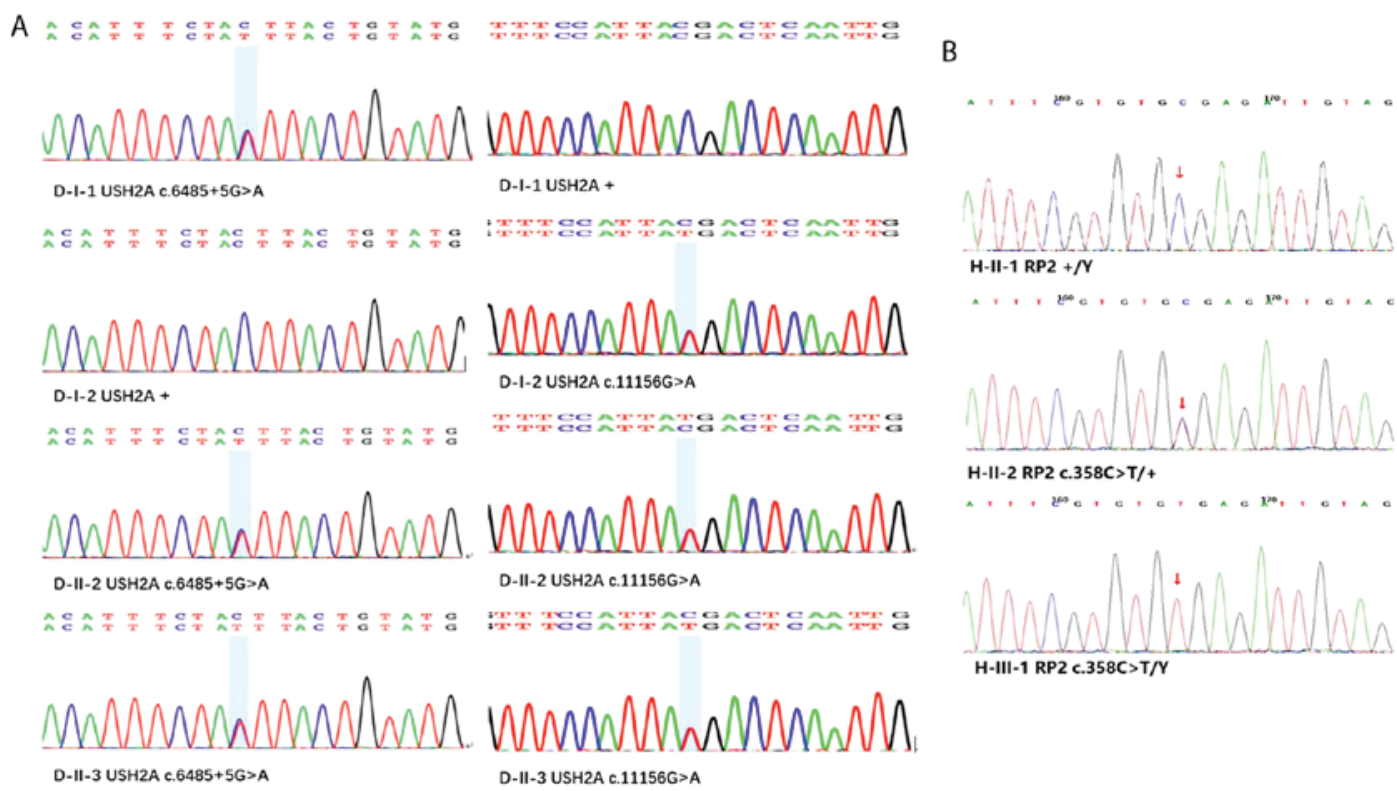

Figure 4. Sanger sequencing. Sanger sequencing results of pathogenic mutations in Families (A) D and (B) H. The blue shaded areas in figure A and red arrows in figure B represent the mutant nucleotide. USH2A, usherin; P2, ARL3 GTPase-activating protein. 
leading to the degeneration of photoreceptors. As the mRNA expression levels of the long splice variant in the cochlea are low, the function of USH2A has been demonstrated to persist in cochlea cells (22). The mutation c.6,485+5G $>\mathrm{A}$ in USH2A was previously reported in a Japanese patient with Usher syndrome type 2 , however, it was only identified in one individual without families involvement (24). In the present study, the two patients shared the same compound mutation in family $\mathrm{H}$, co-segregating with clinical features. In addition, it demonstrated pleiotropy when patients in family $\mathrm{H}$ did not suffer from hearing impairment.

In conclusion, the present study described two Chinese families comprising individuals diagnosed from RP. Through the use of whole-exome sequencing, a compound heterozygous mutation co-segregating with the disease was identified as the pathogenic mutation in ARRP in Family D. To the best of our knowledge, this is the first identification of compound mutation c. $6,485+5 \mathrm{G}>\mathrm{A}$ in $\mathrm{USH} 2 \mathrm{~A}$ as a pathogenic mutation among Chinese patients with RP. However, the pathogenic mechanisms resulting from c. $6,485+5 \mathrm{G}>\mathrm{A}$ require further investigation. In Family H, the XLRP mutation (c.358C $>\mathrm{T}$ ) in RP2 was confirmed to be a pathogenic mutation caused by random $\mathrm{X}$-chromosome inactivation. Future investigations aim to analyze more pedigrees with RP, and examine the pathogenic mechanism of the gene mutation.

\section{Acknowledgements}

The authors are grateful to patients and their family members for the participation.

\section{Funding}

The present study was supported by grants from Shanghai Hospital Development Center (grant no. SHDC12016116) and Renji Hospital (grant no. PYIII-17-030).

\section{Availability of data and materials}

The datasets used and analyzed during the present study are available from the corresponding author on reasonable request.

\section{Authors' contributions}

YF contributed to conception and design of the study, blood sample collection, data acquisition, statistical analysis, data interpretation and drafting of the manuscript; $\mathrm{NC}$ contributed to data acquisition, data interpretation and critical revision of the manuscript for scientific and factual content; ZQ contributed to critical revision of the design of the study; JS contributed to supervision, conception and design of the study, data interpretation and critical revision of the manuscript; LL contributed to supervision, conception and design of the study and critical revision of the manuscript.

\section{Ethics approval and consent to participate}

The present study was approved by the Ethics Committee of Ren Ji Hospital [reference number (2016) 219 (2)]. Informed consent was obtained from all the patients involved.

\section{Patient consent for publication}

Not applicable.

\section{Competing interests}

The authors declare that they have no competing interests.

\section{References}

1. Pawlyk BS, Bulgakov OV, Sun X, Adamian M, Shu X, Smith AJ, Berson EL, Ali RR, Khani S, Wright AF, et al: Photoreceptor rescue by an abbreviated human RPGR gene in a murine model of X-linked retinitis pigmentosa. Gene Ther 23: 196-204, 2016.

2. Dryja TP, Hahn LB, Kajiwara K and Berson EL: Dominant and digenic mutations in the peripherin/RDS and ROM1 genes in retinitis pigmentosa. Invest Ophthalmol Vis Sci 38: 1972-1982, 1997.

3. Holt IJ, Harding AE, Petty RK and Morgan-Hughes JA: A new mitochondrial disease associated with mitochondrial DNA heteroplasmy. Am J Hum Genet 46: 428-433, 1990.

4. Benjaminy S, Kowal SP, MacDonald IM and Bubela T: Communicating the promise for ocular gene therapies: Challenges and recommendations. Am J Ophthalmol 160: 408-415.e2, 2015.

5. Di Y, Huang L, Sundaresan P, Li S, Kim R, Ballav Saikia B, Qu C, Zhu X, Zhou Y, Jiang Z, et al: Whole-exome sequencing analysis identifies mutations in the EYS gene in retinitis pigmentosa in the Indian population. Sci Rep 6: 19432, 2016.

6. Avila-Fernandez A, Perez-Carro R, Corton M, Lopez-Molina MI, Campello L, Garanto A, Fernandez-Sanchez L, Duijkers L, Lopez-Martinez MA, Riveiro-Alvarez R, et al: Whole-exome sequencing reveals ZNF408 as a new gene associated with autosomal recessive retinitis pigmentosa with vitreal alterations. Hum Mol Genet 24: 4037-4048, 2015.

7. Li H and Durbin R: Fast and accurate long-read alignment with Burrows-Wheeler transform. Bioinformatics 26: 589-595, 2010.

8. Lan X, Gao H, Wang F, Feng J, Bai J, Zhao P, Cao L, Gui S, Gong L and Zhang Y: Whole-exome sequencing identifies variants in invasive pituitary adenomas. Oncol Lett 12: 2319-2328, 2016.

9. DePristo MA, Banks E, Poplin R, Garimella KV, Maguire JR, Hartl C, Philippakis AA, del Angel G, Rivas MA, Hanna M, et al: A framework for variation discovery and genotyping using next-generation DNA sequencing data. Nat Genet 43: 491-498, 2011.

10. McKenna A, Hanna M, Banks E, Sivachenko A, Cibulskis K, Kernytsky A, Garimella K, Altshuler D, Gabriel S, Daly M and DePristo MA: The genome analysis Toolkit: A MapReduce framework for analyzing next-generation DNA sequencing data. Genome Res 20: 1297-1303, 2010.

11. Van der Auwera GA, Carneiro MO, Hartl C, Poplin R, Del Angel G, Levy-Moonshine A, Jordan T, Shakir K, Roazen D, Thibault J, et al: From FastQ data to high confidence variant calls: The genome analysis Toolkit best practices pipeline. Curr Protoc Bioinformatics 43: 11.10.1-33, 2013.

12. Abyzov A, Urban AE, Snyder M and Gerstein M: CNVnator: An approach to discover, genotype, and characterize typical and atypical CNVs from family and population genome sequencing. Genome Res 21: 974-984, 2011.

13. Evans RJ, Chapple JP, Grayson C, Hardcastle AJ and Cheetham ME: Assay and functional analysis of the ARL3 effector RP2 involved in X-linked retinitis pigmentosa. Methods Enzymol 404: 468-480, 2005.

14. Kuhnel K, Veltel S, Schlichting I and Wittinghofer A: Crystal structure of the human retinitis pigmentosa 2 protein and its interaction with Arl3. Structure 14: 367-378, 2006.

15. Patil SB, Hurd TW, Ghosh AK, Murga-Zamalloa CA and Khanna H: Functional analysis of retinitis pigmentosa 2 (RP2) protein reveals variable pathogenic potential of disease-associated missense variants. PLoS One 6: e21379, 2011.

16. Mears AJ, Gieser L, Yan D, Chen C, Fahrner S, Hiriyanna S, Fujita R, Jacobson SG, Sieving PA and Swaroop A: Protein-truncation mutations in the RP2 gene in a North American cohort of families with X-linked retinitis pigmentosa. Am J Hum Genet 64: 897-900, 1999. 
17. Wutz A: Gene silencing in X-chromosome inactivation: Advances in understanding facultative heterochromatin formation. Nat Rev Genet 12: 542-553, 2011.

18. Fishman GA, Kumar A, Joseph ME, Torok N and Anderson RJ: Usher's syndrome. Ophthalmic and neuro-otologic findings suggesting genetic heterogeneity. Arch Ophthalmol 101: $1367-1374,1983$

19. Jacobson SG, Cideciyan AV, Aleman TS, Sumaroka A, Roman AJ, Gardner LM, Prosser HM, Mishra M, Bech-Hansen NT, Herrera W, et al: Usher syndromes due to MYO7A, PCDH15, USH2A or GPR98 mutations share retinal disease mechanism. Hum Mol Genet 17: 2405-2415, 2008.

20. van Wijk E, Pennings RJ, te Brinke H, Claassen A, Yntema HG, Hoefsloot LH, Cremers FP, Cremers CW and Kremer H: Identification of 51 novel exons of the Usher syndrome type 2A (USH2A) gene that encode multiple conserved functional domains and that are mutated in patients with Usher syndrome type II. Am J Hum Genet 74: 738-744, 2004.

21. Aller E, Sánchez-Sánchez AV, Chicote JU, García-García G, Udaondo P, Cavallé L, Piquer-Gil M, García-España A, Díaz-Llopis M, Millán JM and Mullor JL: Analysis of the Ush2a gene in medaka fish (Oryzias latipes). PLoS One 8: e74995, 2013.
22. Liu X, Bulgakov OV, Darrow KN, Pawlyk B, Adamian M, Liberman MC and Li T: Usherin is required for maintenance of retinal photoreceptors and normal development of cochlear hair cells. Proc Natl Acad Sci USA 104: 4413-4418, 2007.

23. McGee TL, Seyedahmadi BJ, Sweeney MO, Dryja TP and Berson EL: Novel mutations in the long isoform of the USH2A gene in patients with Usher syndrome type II or non-syndromic retinitis pigmentosa. J Med Genet 47: 499-506, 2010

24. Nakanishi H, Ohtsubo M, Iwasaki S, Hotta Y, Mizuta K, Mineta $\mathrm{H}$ and Minoshima S: Identification of 11 novel mutations in USH2A among Japanese patients with Usher syndrome type 2. Clin Genet 76: 383-391, 2009.

This work is licensed under a Creative Commons Attribution-NonCommercial-NoDerivatives 4.0 International (CC BY-NC-ND 4.0) License. 